This is the post peer-review accepted manuscript of:

N. Decarli, F. Guidi and D. Dardari, "Passive UWB RFID for Tag Localization: Architectures and Design," in IEEE Sensors Journal, vol. 16, no. 5, pp. 1385-1397, March1, 2016.

https://doi.org/10.1109/JSEN.2015.2497373

The published version is available online at:

http://ieeexplore.ieee.org/stamp/stamp.jsp?tp=\&arnumber $=7317485$ \&isnumber $=7401148$

(C) 2016 IEEE. Personal use of this material is permitted. Permission from IEEE must be obtained for all other uses, in any current or future media, including reprinting/republishing this material for advertising or promotional purposes, creating new collective works, for resale or redistribution to servers or lists, or reuse of any copyrighted component of this work in other works 


\title{
Passive UWB RFID for Tag Localization: Architectures and Design
}

\author{
Nicolò Decarli Member, IEEE, Francesco Guidi Member, IEEE, and Davide Dardari Senior Member, IEEE
}

\begin{abstract}
In the new scenarios foreseen by the Internet of Things, industrial and commercial systems will be required to detect and localize tagged items with high accuracy, as well as to monitor the level of certain parameters of interest through the deployment of wireless sensors. To meet these challenging requirements, the adoption of passive and semi-passive ultrawideband (UWB) radio-frequency identification (RFID) appears a promising solution which overcomes the limitations of standard Gen.2 ultra-high frequency (UHF) RFID. The design and implementation of such systems pose several practical constraints, impacting the overall network architecture. In this paper, the main issues and challenging aspects for the design of a UWBRFID network considering architectural and protocol choices are discussed in a unitary framework, and practical solutions, accounting for the presented issues, are proposed. Moreover, the possible integration of UWB-RFID with standard Gen.2 UHF-RFID is proposed as an interesting option, discussing architectural solutions, their advantages and drawbacks.
\end{abstract}

Index Terms-RFID, Internet of Things, UWB, sensors, localization.

\section{INTRODUCTION}

$\mathbf{T}$ HE "Internet of Things" (IoT) paradigm is gaining a particular interest for industrial and commercial applications, as it enables a smart connectivity among objects, machines and persons [1]. In this context, the possibility to get real time information from objects and machines, for example, data collected from integrated sensors, in addition to their location in the environment is an important requirement [2], and real-time locating systems (RTLS) represent a candidate to succeed in this goal [3]. Prior to localization, objects need to be identified and, often, some of their properties have to be monitored; to meet this functionality, radio-frequency identification (RFID) systems represent the state-of-the art technology. The increased popularity of these systems derives mainly from the very low cost and extremely low power consumption when passive or semi-passive tags are adopted [4], [5]. The integration of RTLS with RFID will enable new opportunities in terms of joint identification and localization

Manuscript submitted June 15, 2015; revised September 28, 2015; accepted October 19, 2015

N. Decarli and D. Dardari are with the DEI, University of Bologna, Italy (e-mail: \{nicolo.decarli, davide.dardari\}@unibo.it). F. Guidi is with CEA, LETI, MINATEC Campus, 38054 Grenoble, France (e-mail: francesco.guidi@cea.fr).

The associate editor coordinating the review of this paper and approving it for publication was Dr. Amitava Chatterjee.

This research was supported by the Italian Ministerial PRIN project GRETA (Grant 2010WHY5PR), the European H2020 project XCYCLE (Grant 635975), the H2020-EU.1.3.2 IF-EF Marie Curie action MAPS (Grant 659067).

Color versions of one or more of the figures in this paper are available online at http://ieeexplore.ieee.org.

Digital Object Identifier 10.1109/JSEN.2015.2497373

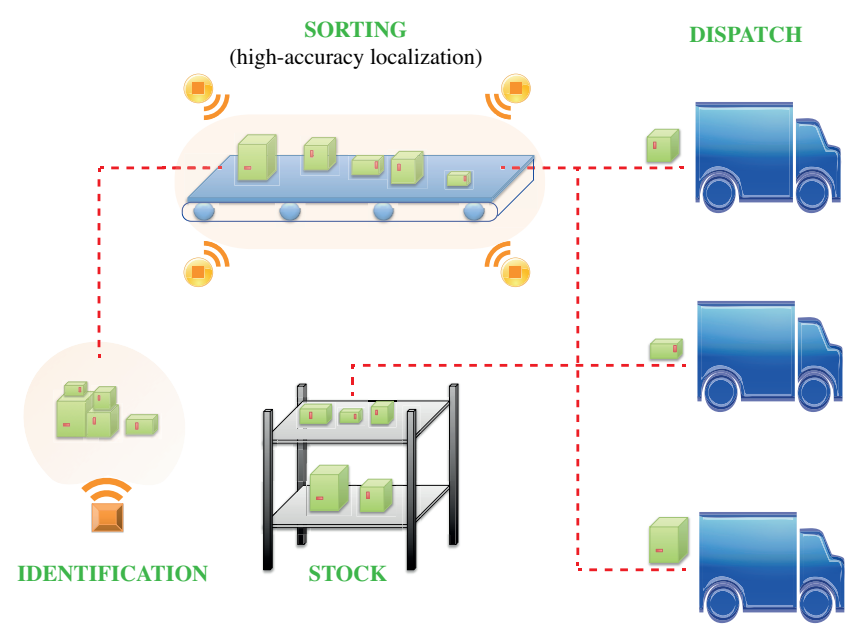

Fig. 1. IoT application example.

and represents one of the most interesting architecture for IoT applications [6].

In many scenarios, different levels of localization accuracy are required: for instance, a few meters for access control (identification), or up to a few centimeters for sorting processes on conveyor belts, as depicted in Fig. 1. While rough localization precision can be obtained with current RFID technology [7]-[9], sub-meter accuracy requires a technology shift. A possibility is represented by the adoption of ultra-wideband (UWB) signals. In fact, the transmission of pulses with the duration in the order of a few nanoseconds guarantees high localization accuracy thanks to accurate time-of-arrival (TOA) estimation [10], [11]. Therefore the joint use of the passive RFID and UWB technologies represents a very appealing solution, and it has been recently proposed [12]. Moreover, additional interest is related to such a technology for two capabilities: (i) the possible integration of sensors to monitor parameters related to the quality of goods or the environment [13]; (ii) the possibility of including in the same network radar functionalities enabling detection of untagged moving persons and objects in the monitored area [6], [14], [15]. Several works have analyzed the joint adoption of UWB and RFID technologies, especially considering active UWB-RFID schemes [16], [17]. For example, in [18], [19] active reflectors at tag side are exploited to reinforce the backscattered signal. Unfortunately, the strict constraints on the energy consumption make solutions based on passive or semi-passive tags more appealing as proposed and investigated in [20]-[22], since tagreader communication is performed by the modulation of the backscatter signal at tag side.

Despite the emerging literature concerning the joint adop- 


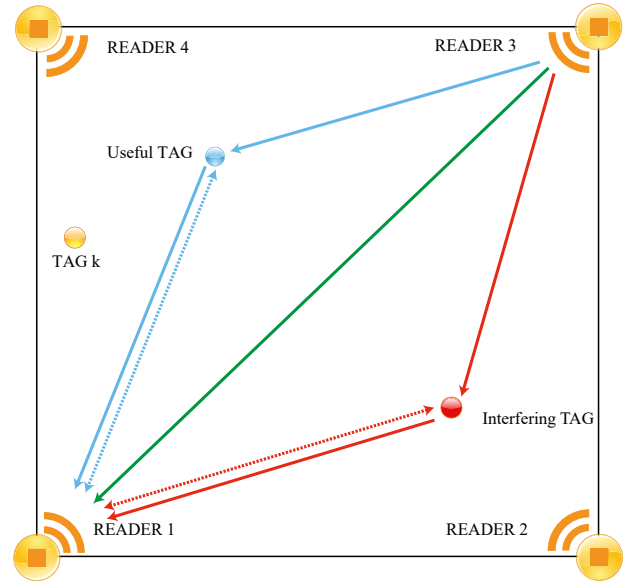

Fig. 2. Reference scenario composed of a square cell monitored by four readers placed at the corners.

tion of UWB and passive RFID technologies, there are no works presenting a global system-perspective of the overall UWB-RFID network architecture and related implementation issues, which significantly differ from ordinary communications and RFID systems. In fact, only practical analyses of specific topics are available. For example, in [21], [23] the UWB backscattering scheme has been analyzed under simplified assumptions, whereas ad hoc prototypes for specific applications are described in [24]. Some papers address only partially the characteristic implementation problems of such architecture and normally consider single-tag operations [22]. For instance, the near-far interference effects and the poor link budget due to the two-hop communication link have been considered, respectively in [25] and [26]. However, their impact on the design of the analog-to-digital converter (ADC) at the receiver has not been addressed yet. In particular, an ad hoc analysis is necessary to assess the detection and demodulation capability of the receiver as a function of the ADC dynamic range and quantization levels when in the presence of clutter and multi-user interference (MUI). In addition, network-related aspects, such as multi-reader deployment and synchronization, have not received any attention, despite their critical effects, as shown in this paper. An effective design of the UWB-RFID network requires all these aspects being accounted simultaneously as they are interlaced each other.

In this paper, we fill in the gap with the previous literature, by presenting in a unitary form a possible and effective design of passive UWB-RFID networks, with particular emphasis on system-related aspects. ${ }^{1}$ The considered UWB-RFID network is composed of readers monitoring an area where tags have to be localized. In particular, tags are semi-passive and based on UWB backscatter modulation, where the low amount of available energy from harvesting or batteries is used only for memory access and to power up the backscatter modulator [12]. ${ }^{2}$ Moreover, in the perspective of a backward

\footnotetext{
${ }^{1}$ Some of the design guidelines here reported have been exploited in the context of the European project SELECT during the system implementation [24].

${ }^{2}$ Tags are not equipped with an active transmitter.
}

compatibility with existing RFID technologies, a completely novel architecture combining the UWB-RFID with today's Gen.2 ultra-high frequency (UHF)-RFID is proposed, and its interesting characteristics for applications in industrial and commercial contexts are presented.

Summarizing, the main contributions of the papers are:

- The review of the main issues and challenging aspects for the realization of a UWB-RFID network with tags based on backscatter modulation, by considering architectural and protocol choices;

- The investigation of design guidelines for tags and readers, accounting for hardware constraints: in particular, the possible reader configurations are analyzed, the synchronization and communication aspects are addressed, and the implementation issues, constraints and innovative solutions are described;

- The introduction of a novel hybrid UHF-UWB RFID architecture, ensuring compatibility with standard Gen.2 UHF-RFID.

The rest of the paper is organized as follows. Sec. II shows the architecture and the signaling scheme considered for the UWB-RFID system. Sec. III describes the main signal processing tasks and revises the problems with the developed solutions. In Sec. IV and Sec. V receiver design and the network deployment issues, respectively, are addressed, starting from the analysis conducted in the previous sections. Sec. VI introduces a new alternative scheme accounting for a tight interaction between the standard Gen.2 UHF-RFID and the novel UWB-RFID, and discusses its various advantages. Finally, Sec. VII concludes the discussion.

\section{System ARChitecture}

A classical RFID scenario is composed of reference nodes, usually referred to as readers and placed in known positions, and tags. Contrarily to tags, readers are active entities capable of transmitting, receiving and processing signals. The goal of the network is to detect the presence of passive tags and estimate their position by analyzing their modulated backscattered response. This modulation is realized by changing the load connected to the tag's antenna [4]. Localization is enabled by the estimation of the signals' propagation time, leading then to distance estimates between readers and tags adopted as input is trilateration-based positioning algorithms (see Sec. V-C).

In this work, the scenario reported in Fig. 2, with the readers at the corners of a square cell, is considered as a reference for the discussion. Obviously, the readers' position can be varied according to the needs, but such placement is assumed as it can guarantee a good coverage of the monitored area. In fact, each tag has to be read by at least three different readers to provide unambiguous localization [3].

\section{A. UWB-RFID Network}

The overall UWB-RFID network architecture comprises a central unit, readers and tags. Each reader communicates with the central unit mostly for transferring the signal processing data (e.g., the TOA estimates allowing the tag localization). In addition, these connections can be exploited to ensure a 


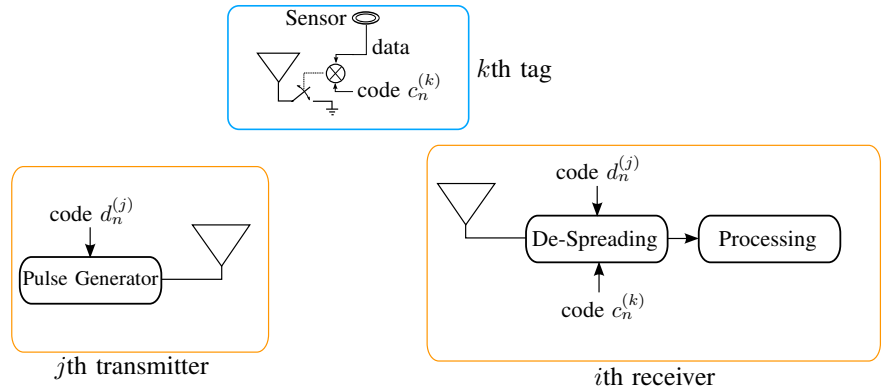

Fig. 3. The considered scheme of the tag, transmitter and receiver.

general coarse synchronization between the readers, as well as for network maintenance. Reader synchronization will be further detailed in Sec. V-B.

Define $\mathcal{T}, \mathcal{E}$ and $\mathcal{R}$ as the set of tags, transmitters and receivers in the environment, respectively, with cardinality $|\mathcal{E}|=N_{\mathrm{t}},|\mathcal{R}|=N_{\mathrm{r}}$ and $|\mathcal{T}|=N_{\mathrm{tag}}$, where tags are placed in unknown positions $\mathbf{p}_{k}=\left(x_{k}, y_{k}\right)$, with $k=1,2, \ldots, N_{\mathrm{tag}}$.

In [6], two different reader configurations are distinguished. In the former, the monostatic network, transmitters are colocated with receivers in known positions $\mathbf{p}_{\mathrm{R} i}=\left(x_{\mathrm{R} i}, y_{\mathrm{R} i}\right)$, with $i=1,2, \ldots, N_{\mathrm{r}}$, and each reader post-processes only the backscattered signal related to its transmitting section. In the latter, the multistatic network, transmitters and receivers, placed in $\mathbf{p}_{\mathrm{T} j}=\left(x_{\mathrm{T} j}, y_{\mathrm{T} j}\right)$ and $\mathbf{p}_{\mathrm{R} i}=\left(x_{\mathrm{R} i}, y_{\mathrm{R} i}\right)$, respectively, with $j=1,2, \ldots, N_{\mathrm{t}}$, and $i=1,2, \ldots, N_{\mathrm{r}}$, are not necessarily co-located. In fact, each receiver can post-process the backscattered signal related to other transmitters. Note that while in traditional RFID systems readers are usually in monostatic configuration, here transmitters and receivers can be also separated. Then, synchronization with a separated transmitter has to be addressed (see Sec. V-B). The two solutions may be also combined in hybrid architectures, where a receiver listens to a subset of the transmitted interrogation signals. In the remainder of the paper it is referred to as reader the reference node composed of at least a receiver. Recently, practical advantages of multistatic configurations for RFID networks, including enhanced localization accuracy and interference rejection, have been highlighted [6], [27], [28].

\section{B. Signaling Scheme}

Several signal processing tasks have to be accomplished by the readers in order to provide information about the tags to the network. The first task consists of tag detection, that is the process to determine the presence of a tag in a monitored area. Secondly, the reader has to perform TOA estimation, thus enabling localization capabilities by fusing at the central unit several tag observations. Due to the low complexity of the tag and to the fact that tags cannot directly communicate, no cooperative techniques can be exploited for performance improvement and coverage extension, so every point of the monitored area must be directly covered by a sufficient number of readers. Finally, if the tag itself contains data to be transmitted to the reader (e.g., because it has an embedded sensor, or data related to the object to which it is attached), the receiver has to perform signal demodulation.

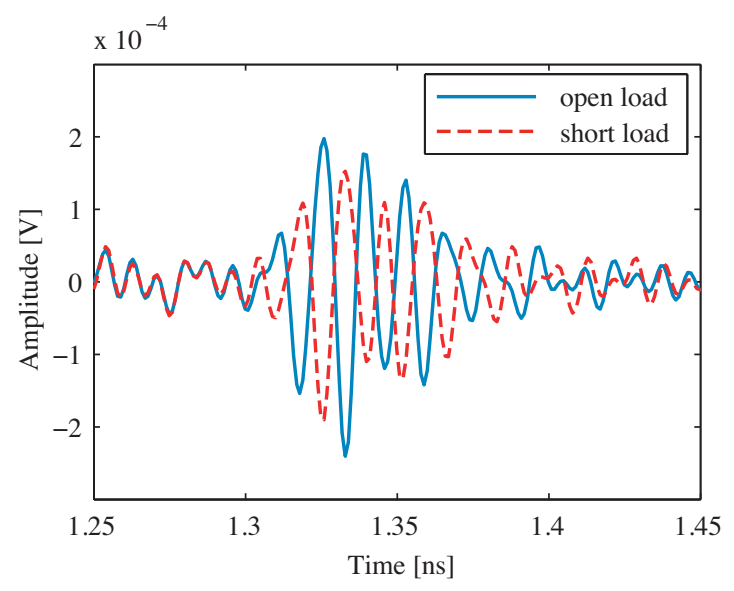

Fig. 4. Example of the backscattered signal for tag open and short circuit loads (only the antenna mode component is shown).

In the following discussion, concerning the processing requirements previously listed, consider the scenario of Fig. 2, where each reader interrogates $N_{\text {tag }}$ tags located in the area. In Fig. 3 the architectures for tag and reader, with separated transmitter and receiver, are shown. During the interrogation phase, the $j$ th transmitter sends an UWB interrogation signal composed of symbols of duration $T_{\mathrm{s}}$, each given by

$$
\begin{aligned}
s_{\mathrm{T} j}(t) & =\sum_{m=0}^{N_{\mathrm{p}}-1} \sum_{n=0}^{N_{\mathrm{c}}-1} d_{n}^{(j)} g\left(t-n T_{\mathrm{c}}-m T_{\mathrm{s}}\right) \\
& =\sum_{m=0}^{N_{\mathrm{p}}-1} \sum_{n=0}^{N_{\mathrm{c}}-1} d_{n}^{(j)} \sum_{i=0}^{N_{\mathrm{pc}}-1} p\left(t-n T_{\mathrm{c}}-i T_{\mathrm{p}}-m T_{\mathrm{s}}\right)
\end{aligned}
$$

where $\left\{d_{n}^{(j)}\right\}$ is the $j$ th transmitter's code, $N_{\mathrm{c}}, N_{\mathrm{pc}}, N_{\mathrm{p}}$, are the number of chips (code length), pulses per chip and transmitted symbols, respectively, $T_{\mathrm{c}}$ and $T_{\mathrm{s}}$ are the chip and symbol time, respectively, and $p(t)$ is the transmitted UWB pulse, repeated with pulse repetition period (PRP) $T_{\mathrm{p}}$.

The tag switches its backscatter modulator status at each chip time $T_{\mathrm{c}}$, according to an antipodal binary code $\left\{c_{n}^{(k)}\right\}$, for $n=0,1, \ldots, N_{\mathrm{c}}-1$, allowing the creation of a unique backscattered channel for each tag. An example of UWB backscattered signal corresponding to two different antenna load configurations (open/short) is shown in Fig. 4, when a single UWB pulse is emitted by the reader. As can be noticed, there is a polarity change in the reflected antenna mode component, which is the only part depending on the antenna load, and that can be exploited for tag identification and localization with the signal processing herein described. ${ }^{3}$ Considering $N_{\mathrm{p}}$ transmitted symbols, the backscatter modulator signal commanding the switch of the $k$ th tag is

$$
m^{(k)}(t)=\sum_{m=0}^{N_{\mathrm{p}}-1} b_{m}^{(k)} \sum_{n=0}^{N_{\mathrm{c}}-1} c_{n}^{(k)} \cdot \Pi\left(\frac{1}{T_{\mathrm{c}}}\left[t-m T_{\mathrm{s}}-n T_{\mathrm{c}}\right]\right)
$$

\footnotetext{
${ }^{3}$ We refer the reader to [20], [29]-[31] for a detailed electromagnetic analysis of UWB backscattering and antenna layouts.
} 
with $\Pi(t)$ denoting the rectangular function of unitary duration for $t \in[0,1]$. In this way, the polarity of the backscattered signal changes at each chip time $T_{\mathrm{c}}$ according to the $k$ th tag's code value $c_{n}^{(k)}$, with $k \in \mathcal{T}$, while the polarity of the whole symbol composed of $N_{\mathrm{s}}$ pulses is modulated according to the data bit $b_{m}^{(k)} \in\{ \pm 1\}$ transmitted from the tag to the reader [32].

Each interrogation signal is backscattered by the tags as well as by the surrounding scatterers of the environment. Specifically, the signal at the $i$ th receiver due to the signal transmitted by the $j$ th transmitter, can be written as

$$
\breve{r}_{i, j}(t)=r_{i, j}^{\mathcal{T}}(t)+r_{i, j}^{\mathcal{S}}(t)+n(t)
$$

where $r_{i, j}^{\mathcal{T}}(t)$ denotes the tags contribution ${ }^{4}$ and $r_{i, j}^{\mathcal{S}}(t)$ accounts for the static contribution (comprehensive of multipath) between transmitter and receiver. ${ }^{5}$ Finally, $n(t)$ models the additive white Gaussian noise (AWGN) with one-sided power spectral density $N_{0}$.

The tags' contribution is given by ${ }^{6}$

$$
r_{i, j}^{\mathcal{T}}(t)=\sum_{k \in \mathcal{T}}\left[\left(s_{\mathrm{T} j}(t) \otimes h_{\mathrm{down}}^{(j, k)}(t)\right) \cdot m^{(k)}(t)\right] \otimes h_{\mathrm{up}}^{(k, i)}(t)
$$

where $h_{\text {down }}^{(j, k)}(t)$ is the downlink channel impulse response (CIR), de-embedded of the propagation time, related to the link $j$ th transmitter - $k$ th tag, $h_{\mathrm{up}}^{(k, i)}(t)$ is the uplink CIR related to the link $k$ th tag - $i$ th receiver.

It is possible to rearrange (4) as

$r_{i, j}^{\mathcal{T}}(t)=\sum_{k \in \mathcal{T}} \sum_{m=0}^{N_{\mathrm{p}}-1} \sum_{n=0}^{N_{\mathrm{c}}-1} d_{n}^{(j)} c_{n}^{(k)} b_{m}^{(k)} w_{i, j, k}^{\mathcal{T}}\left(t-m T_{\mathrm{s}}-n T_{\mathrm{c}}-\tau_{i, j, k}\right)$

where $\tau_{i, j, k}$ is the signal TOA, considered with respect to the transmitter's clock, and $w_{i, j, k}^{\mathcal{T}}(t)=g(t) \otimes h_{\text {down }}^{(j, k)}(t) \otimes h_{\mathrm{up}}^{(k, i)}(t)$ is the channel response to $g(t)$ for the $k$ th tag. Specifically, it is $\tau_{i, j, k}=\left(d_{\mathrm{T} j}+d_{\mathrm{R} i}\right) / c$, where $d_{\mathrm{T} j}$ is the distance between the $j$ th transmitter and the tag, $d_{\mathrm{R} i}$ is the distance between the tag and the $i$ th receiver, ${ }^{7}$ and $c$ denotes the speed of light.

Note that the round trip backscattering channel is strongly unfavorable from the energetic point of view, since the received backscattered signal experiences twice the path loss between the reader and the $\operatorname{tag}^{8}$ [35]-[37]. In fact, the distancedependence of the received signal power scales, in free space, with the fourth power of the reader-tag distance, resulting in a maximum reading distance much smaller than for an ordinary one-hop communication link [38]. It is important to underline that the tag backscattering behavior is impacted by the presence of the object on which it is attached. However, differently from standard UHF-RFID, the typical materials which constitute the objects where tags are attached to do

\footnotetext{
${ }^{4}$ Here only the tag's antenna mode component is included [12].

${ }^{5} \mathrm{We}$ neglect the presence of fast moving objects and tags in the environment, as well as the effects of diffuse clutter [6], [33].

${ }^{6}$ Operator $\otimes$ denotes the convolution.

${ }^{7}$ Both $d_{\mathrm{T} j}$ and $d_{\mathrm{R} i}$ are function of the tag index $k$, however this dependence has been omitted for notation convenience.

${ }^{8}$ The effect is more accentuated due to the classical carrier frequency around $4 \mathrm{GHz}$ usually adopted for UWB signals [34].
}

not detune significantly the tag thanks to the large bandwidth adopted [39].

Together with the tag response, the receivers collect the signal reflected by the surrounding environment, which composes the static contribution (clutter component). Such a static contribution $r_{i, j}^{\mathcal{S}}(t)$ in (3) is

$$
r_{i, j}^{\mathcal{S}}(t)=s_{\mathrm{T} j}(t) \otimes h_{\mathrm{c}}^{(j, i)}(t)
$$

having indicated with $h_{\mathrm{c}}^{(j, i)}(t)$ the CIR of the link between the $j$ th transmitter and the $i$ th receiver, independent of tags' backscattering. As before, (6) can be reformulated as

$$
r_{i, j}^{\mathcal{S}}(t)=\sum_{m=0}^{N_{\mathrm{p}}-1} \sum_{n=0}^{N_{\mathrm{c}}-1} d_{n}^{(j)} w_{i, j}^{\mathcal{S}}\left(t-m T_{\mathrm{s}}-n T_{\mathrm{c}}-\tau_{i, j}\right)
$$

where $\tau_{i, j}$ is the signal TOA, considered with respect to the transmitter's clock, and $w_{i, j}^{\mathcal{S}}(t)=g(t) \otimes h_{\mathrm{c}}^{(i, j)}(t)$ is the channel response to $g(t)$ for the static contribution. Specifically it is $\tau_{i, j}=d_{i, j} / c$, where $d_{i, j}$ is the distance between the $j$ th transmitter and the $i$ th receiver.

Discrimination between the useful contribution backscattered from the $k$ th tag and those reflected by other tags and the direct transmitter-to-receiver signal is ensured by a despreading procedure at the receiver (see Fig. 3). Specifically, the separation is enabled by the adoption of different spreading codes, since the tag signals are modulated according to the composed code $\left\{d_{n}^{(j)} \cdot c_{n}^{(k)}\right\}$, while the static contribution independent of tags' backscattering is modulated according to the transmitter code $\left\{d_{n}^{(j)}\right\}$ only [6]. In the following, starting from the signaling scheme here described, the management of the tags in the scenario is described.

\section{TAG MANAGEMENT}

When multiple UWB tags based on backscatter modulation are deployed in the environment, several aspects have to be accounted. Here the solutions developed for tag management are reported in a unitary form, in order to drive the subsequent discussion about the receiver design and the network deployment of Sec. IV and Sec. V.

\section{A. Tag Synchronization}

Tag synchronization is a crucial operation in the UWBRFID systems. In fact, if code generators of tags are completely free running, the reader must perform an exhaustive code acquisition search in order to synchronize its local code generator, used for the de-spreading, with that of the intended useful tag to be detected. In addition, the level of readertag synchronization imposes constraints on the tag codes assignment to counteract MUI, as detailed in Sec. III-C. To accomplish this task, an additional narrowband link, for example in the UHF band or in the $2.4 \mathrm{GHz}$ band, can be used to derive the synchronization signal necessary to wake-up tags, initially in an idle state, and to reset the tags' spreading code generators. The same wake-up signal can also be used to energize the tag by exploiting energy harvesting techniques, thus making the tag fully passive, that is, energy autonomous. In fact, due to the stringent emission limits on the UWB 


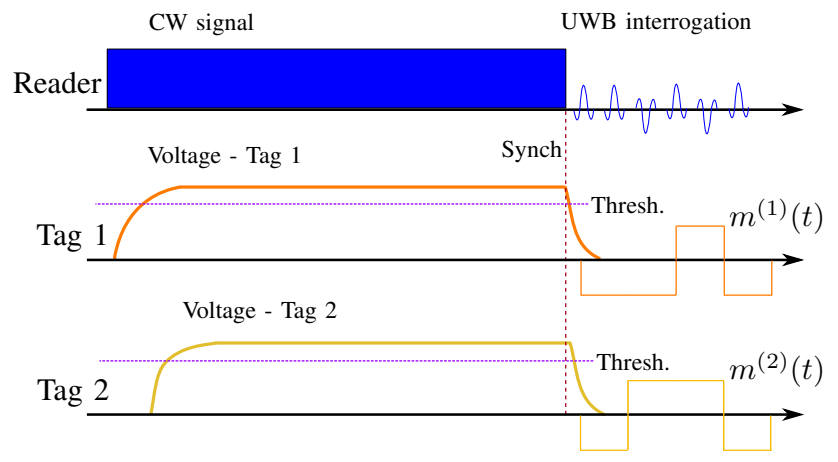

Fig. 5. Wake-up synchronization scheme and signaling.

mask, no significant energy can be extracted by the UWB interrogation signals of readers to power up the tags.

Among the various possibilities, an interesting solution is to perform the reset of the tag code generator on the falling edge of a wake-up continuous wave (CW) carrier received by tags, as shown in Fig. 5. Note that the $\mathrm{CW}$ powers up tags, by charging a capacitor via the antenna and a rectifier circuit. Once the $\mathrm{CW}$ signal has ended, the falling of the resulting voltage is used for initiating the backscatter modulation of the UWB signals. In this way the propagation-dependent capacitor charge time ${ }^{9}$ does not play a significant role in the synchronization jitter, since the discharge starting event is not affected by the path loss and depends only on the tags' circuitry. Such solutions have been proposed and experimented in [19], [40]. The maximum offset after this procedure is expected to be in the order of a few microseconds. Moreover, if the narrowband link exploited for wake-up is modulated with a proper signal (e.g., exploiting amplitude shift keying (ASK)/on-off keying (OOK) modulations), the demodulated signal at tag side can serve as reference to lock the local tag oscillator, and helps to prevent the clock drift effects [25]. Such clock drifts could be present at tag side due to the expected poor characteristics of the low-cost oscillators driving the backscatter modulator. ${ }^{10}$ The clock drift determines a time slide between the modulation signal in the tag and the despreading at the reader that might compromise the correct detection of the signal. The longer is the duration of the UWB packet, that is, $N_{\mathrm{p}} T_{\mathrm{s}}$, the more the drift effect is detrimental.

\section{B. Tag Detection}

Once tags are synchronized, the UWB reader-tag communication begins, since tags start modulating the transmitted pulses that are successively received by the readers (see Fig. 5).

As described in Sec. II-B, tag multiple access is realized thanks to the assignment of different spreading codes to tags. In this case, since we are in presence of a code division multiple access (CDMA) and each spreading code is uniquely assigned to a tag, a decision at the output of the de-spreading

\footnotetext{
${ }^{9}$ This is due to the narrowband CW signals that may experience selectively channels, and to different reader-tag distances.

${ }^{10}$ An example of ad-hoc oscillator for tags based on UWB backscattering has been presented in [41].
}

phase is sufficient to accomplish the identification purpose. De-spreading is operated, as described in [32], by accumulating the responses of the $N_{\mathrm{s}}$ pulses that compose a symbol. In particular, each response of the channel to a transmitted pulse is multiplied at receiver side by the code element $\tilde{d}_{n}^{(j)}$ of the intended transmitter and the code element $\tilde{c}_{n}^{(k)}$ of the intended tag, and then summed up to the others composing a symbol. Codes $\left\{\tilde{d}_{n}^{(j)}\right\}$ and $\left\{\tilde{c}_{n}^{(k)}\right\}$ denote the periodically repeated sequences of period $N_{\mathrm{s}}=N_{\mathrm{c}} N_{\mathrm{pc}}$ as $\tilde{c}_{n}^{(k)} \triangleq c_{\left\lfloor n / N_{\mathrm{pc}}\right\rfloor}^{(k)}$ and $\tilde{d}_{n} \triangleq d_{\left\lfloor n / N_{\mathrm{pc}}\right\rfloor}^{(j)}$ for $n=0,1, \ldots, N_{\mathrm{s}}-1$, with $\tilde{c}_{n+N_{\mathrm{s}}}^{(k)}=\tilde{c}_{n}^{(k)}$, $\tilde{d}_{n+N_{\mathrm{s}}}^{(j)}=\tilde{d}_{n}^{(j)}$, where $\lfloor\cdot\rfloor$ indicates the floor operation. Such a de-spreading operation allows isolating the signals related to a specific transmitter-tag pair from clutter and interference, and produces a processing gain counteracting the receiver noise [32].

The optimal processing technique requires a matched filter [32] but its implementation is often too complex due to the large bandwidth so energy-based detection techniques are usually considered [21]. Generically, tag detection is realized at each reader by checking if the de-spreading output level related to a specific tag code is above a certain threshold. When more than one reader is tuned to the same tag, the decision on the tag presence can be taken by properly combining the different observations in the central unit. The detection and demodulation of multiple tags requires the replication of the same receiver structure, with multiple de-spreaders each tuned to a specific tag code. Alternatively, the same de-spreader can be re-used for different tags in different interrogation cycles, changing properly the tag code, at the expense of the refresh rate, that is the capability of the system of offering a new reading of a specific tag.

It is important to underline that even if tag detection has been properly performed, the receiver has to continue track a certain tag code to follow the tag clock drift. Note that in UWB backscattering, the clock drift at tag side does not affect the TOA of the backscattered pulse (which is determined only by the transmitter, supposed to be equipped with a highaccuracy clock), but it affects the pulses modulation operated by spreading codes. In addition to detection, demodulation of the bits $\left\{b_{m}^{(k)}\right\}$ allows data communication between tags and readers. In this manner the tag ID can be transferred to the network or, if the tag has embedded sensors, measurements can be transmitted. Data modulation can be accomplished via binary phase shift keying (BPSK) modulation of the overall sequence of $N_{\mathrm{s}}$ pulses composing a symbol, as described in [32].

In order to have an idea of potential operating ranges, Table I shows the number of pulses $N_{\mathrm{s}}$ required to ensure a theoretical reader-tag distance, in monostatic configuration, when an energy detector receiver is considered, for a signalto-noise ratio (SNR) which let to guarantee a probability of false alarm $10^{-3}$ and a probability of detection 0.9 , under the European mask constraint [21]. In the same table the maximum refresh rate when a packet of 128 bits is transmitted 
TABLE I

MiNimum NUMBER OF PULSES FOR A TARGET READER-TAG READING RANGE AND REFRESH RATE WITH A 128 BIT PACKET TRANSMISSION.

\begin{tabular}{|c|c|c|c|c|c|c|c|c|c|c|}
\hline Reader-Tag distance & $1 \mathrm{~m}$ & $2 \mathrm{~m}$ & $3 \mathrm{~m}$ & $4 \mathrm{~m}$ & $5 \mathrm{~m}$ & $6 \mathrm{~m}$ & $7 \mathrm{~m}$ & $8 \mathrm{~m}$ & $9 \mathrm{~m}$ & $10 \mathrm{~m}$ \\
\hline$N_{\mathrm{S}}$ & 6 & 88 & 441 & 1393 & 3400 & 7049 & 13059 & 22277 & 35683 & 54387 \\
Refresh Rate & $10173 \mathrm{~Hz}$ & $694 \mathrm{~Hz}$ & $138 \mathrm{~Hz}$ & $44 \mathrm{~Hz}$ & $18 \mathrm{~Hz}$ & $9 \mathrm{~Hz}$ & $5 \mathrm{~Hz}$ & $3 \mathrm{~Hz}$ & $2 \mathrm{~Hz}$ & $1 \mathrm{~Hz}$ \\
\hline
\end{tabular}

TABLE II

SIMULATION PARAMETERS.

\begin{tabular}{|c|c|c|}
\hline Parameter & Symbol & Value \\
\hline EIRP & $P_{\mathrm{T}}$ & $-11 \mathrm{dBm}$ \\
Bandwidth & $W$ & $1.5 \mathrm{GHz}$ \\
Center frequency & $f_{\mathrm{c}}$ & $4 \mathrm{GHz}$ \\
Pulse interval & $T_{\mathrm{p}}$ & $128 \mathrm{~ns}$ \\
Reader antenna gain & $G_{\mathrm{r}}$ & $5 \mathrm{dBi}$ \\
Tag antenna gain & $G_{\mathrm{t}}$ & $1 \mathrm{dBi}$ \\
Tag losses & $L_{\mathrm{t}}$ & $2 \mathrm{~dB}$ \\
Pulses per symbol & $N_{\mathrm{s}}$ & 32768 \\
Receiver noise figure & $F$ & $4 \mathrm{~dB}$ \\
Signal-to-Noise Ratio & $\mathrm{SNR}$ & $13 \mathrm{~dB}$ \\
\hline
\end{tabular}

from the tag to the reader is also shown. ${ }^{11}$ Notice that the refresh rate shown in Table I can be significantly increased if a code $c_{n}^{(k)}$ is uniquely assigned to a tag. In fact, in this case, there is direct correspondence between the UWB spreading code and the tag ID, so tag detection is sufficient for providing to the reader the tag identity. Here and in the following of the paper, results have been obtained considering the system parameters reported in Table II. It is important to underline that the larger is $N_{\mathrm{s}}$ (i.e., the symbol time), the longer will be the maximum operating reader-tag distance, thanks to an increasing processing gain at receiver side. As reported in Sec. II-B, the number of pulses per symbol $N_{\mathrm{s}}$ is determined by both the code length $N_{\mathrm{c}}$ and by the number of pulses per chip $N_{\mathrm{pc}}$. In general, increasing the number of pulses per chip, with a fixed PRP, decreases the energy consumption since the tag average switching rate between open and short circuit is decremented. Furthermore, as described in [21], this helps on avoiding detrimental effects due to synchronization mismatches between tags and receivers.

\section{Multi-Tag Interference}

With the proposed CDMA scheme, all tags present in the monitored area simultaneously respond to the reader interrogation, without the need of any anti-collision protocol as required in the EPC Gen.2 RFID standard [42], [43]. Such an access technique poses particular constraints in terms of code assignment to tags. The behavior of the adopted codes, and the degree of synchronization between readers and tags, determine the amount of interference at the receiver. Such interference may produce false alarms during the detection of a desired tag [25] and worsen the demodulation performance [32]. Moreover, due to the two-hop propagation link characteristic of backscatter propagation, near-far interference effects can drastically affect the performance; in fact, a tag

\footnotetext{
${ }^{11}$ The number of bits has been chosen larger than the standard 96 bits of the electronic product code (EPC) provided by the Gen.2 UHF-RFID to account for possible sensor data and parity.
}

close to a reader can produce an irreducible interference on the detector output of a farer tag, preventing the possibility of a correct detection. An extensive analysis of the code assignment strategies for tags and an overview of their design guidelines is reported in [21]. We want here to stress the importance of adopting balanced, or quasi-balanced, codes (i.e., codes with zero mean value) that allow the clutter cancellation at receiver side [32], as will be discussed in the next section.

A main limitation of the analyzed CDMA scheme is represented by the necessity of providing each tag with a unique spreading code. Table III reports the number of different available codewords for several code families of interest for the UWB-RFID system, considering typical codes lengths. As example, a good choice allowing a substantial tradeoff between interference mitigation, complexity and detection performance is $N_{\mathrm{c}}=128$, as shown in [21]. In this case, adopting balanced Gold codes, which represent good candidates, only 65 different tags can be managed. Note that, in case of good reader-tag synchronization, the same spreading code can be assigned to several tags with a different initial phase shift, providing that this is greater than the possible level of asynchronism of the system. In this manner the number of manageable tags is increased. A new alternative solution capable of overcoming the limitation on the number of different tags will be introduced in Sec. VI.

\section{Clutter Removal}

The RFID-UWB system suffers from clutter that is determined by the environmental response not depending on tag's backscattering [12]. In monostatic networks the clutter includes the transmitting-receiving antenna coupling, which can be avoided with time-gating operations on the received signal [22], resulting in blind zones around the reader where the tag cannot be detected. Another clutter contribution is given by the tag's structural mode, which is the backscattered component independent of the tag's antenna load. In this case, it has been proposed to separate in the time-domain the structural and the antenna mode by adopting a delay line at tag side between the antenna port and the backscattering modulator [22], [30]. The last clutter contribution is given by the response of the environment. The ensemble of all these signals determines the static contribution $r_{i, j}^{\mathcal{S}}(t)$ at receiver side.

The clutter component must be properly canceled in order to provide robust tag detection. The proposed CDMA approach is intrinsically immune to clutter if balanced codes are adopted at tag side, regardless of the reader's code [6], [32]. In fact, with the de-spreading, each static response to a transmitted pulse is multiplied for the tag code $c_{n}^{(k)}$ and accumulated; the balanced tag code makes then null the clutter output 
TABLE III

NUMBER OF AVAILABLE CODEWORDS FOR DIFFERENT CODE FAMILIES.

\begin{tabular}{|c|c|c|c|c|c|c|c|c|c|c|}
\hline$N_{\mathrm{C}}$ & $15 / 16$ & $31 / 32$ & $63 / 64$ & $127 / 128$ & $255 / 256$ & $511 / 512$ & $1024 / 1024$ & $2047 / 2048$ & $4095 / 4096$ & $8191 / 8192$ \\
\hline Orthogonal (balanced) & 15 & 31 & 63 & 127 & 255 & 511 & 1023 & 2047 & 4095 & 8191 \\
\hline M-sequences & 2 & 6 & 6 & 18 & 16 & 48 & 60 & 176 & 144 & 630 \\
\hline Gold (balanced) & - & 17 & 33 & 65 & - & 257 & 513 & 1025 & - & 4097 \\
\hline
\end{tabular}

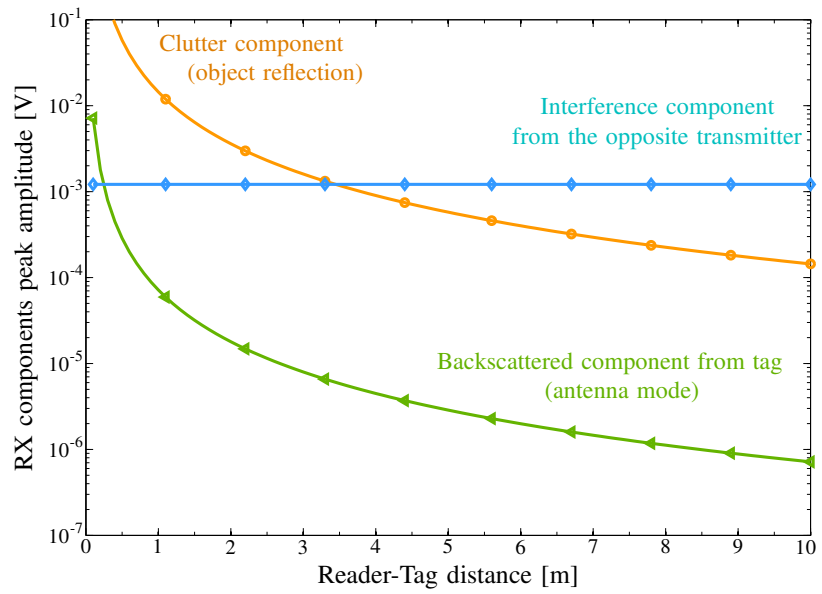

Fig. 6. Typical dynamic range of a UWB-RFID system.

contribution after the accumulation of the $N_{\mathrm{s}}$ pulses. Alternative solutions for clutter suppression deal with classical radar signal processing, such as filtering techniques that remove the estimated background response when the tag is absent [22]. The main drawbacks are represented by the necessity of channel estimation and very high-speed signal processing, additionally with severe limitations in terms of tag movement.

From the implementation point of view, one of the main challenges is the digital removal of the clutter component, whose level is usually several orders of magnitude higher than signals from tags, as will be studied in Sec. IV-A, thus conditioning the required dynamic range at the receiver.

\section{RECEIVER DESIGN}

In the previous sections, a survey of the main challenges related to the design of the passive UWB-RFID system was presented. Now, starting from the issues and constraints underlined, the main guidelines for the receiver design will be derived, based also on so-far unexplored reader configurations for RFID applications. Note that such a design must be carefully carried out due to the specificity of the considered system where different signal components are present at the reader input. In fact, as will be investigated, the signals of interest coming from tags are dominated by the various static contributions (i.e., the clutter), and the receiver must properly handle such weak signals preventing ADC saturation due to the strongest components.

\section{A. Receiver Dynamic Range}

In the practical implementation of the UWB receiver it is important to account for the relative levels of the received signals, both useful and interfering, in order to reduce the possibility of ADC saturation and poor quantization. For this reason, the signal characteristics expected at the receiving antenna port are now analyzed. Specifically, the received signal is composed of the following components:

1) The signals backscattered by the tags, related to the interrogation of a specific transmitter, which the reader wants to demodulate;

2) The signals backscattered by the tags related to the interrogation signal coming from other transmitters (i.e., an interference component);

3) The interference of other transmitters, that is, the ensemble of signals emitted by other transmitters independent of tags' backscattering;

4) The signal emitted by a co-located transmitter and reflected by the environment, independent of tags' backscattering.

The first two components are the tags' contribution $r_{i, j}^{\mathcal{T}}(t)$; the last two components are the static contribution (i.e., clutter) $r_{i, j}^{\mathcal{S}}(t)$ (see Sec. II-B). For the sake of a complete characterization of the dynamic range, it is important to analyze the expected amplitude of these signals, as well as the ratio between the strongest input signal and the signal backscattered by the intended tag (useful tag), which is supposed to be strongly attenuated by the two-way channel.

Assuming for simplicity a monostatic configuration, the path loss related to the different components of the received signal, obtained adopting the free-space propagation model at a single central frequency, can be written as

$$
\begin{aligned}
\mathrm{PL}_{i, k}^{(1,2)} & =\left[\frac{G_{\mathrm{r}}^{2} G_{\mathrm{t}}^{2}}{L_{\mathrm{t}}}\left(\frac{\lambda}{4 \pi\left|\mathbf{p}_{\mathrm{R} i}-\mathbf{p}_{k}\right|}\right)^{4}\right]^{-1} \\
\mathrm{PL}_{i, j}^{(3)} & =\left[G_{\mathrm{r}}^{2}\left(\frac{\lambda}{4 \pi\left|\mathbf{p}_{\mathrm{T} j}-\mathbf{p}_{\mathrm{R} i}\right|}\right)^{2}\right]^{-1} \\
\mathrm{PL}_{i, k}^{(4)} & =\left[\sigma G_{\mathrm{r}}^{2} \frac{\lambda^{2}}{(4 \pi)^{3}}\left(\frac{1}{\left|\mathbf{p}_{\mathrm{R} i}-\mathbf{p}_{k}\right|}\right)^{4}\right]^{-1}
\end{aligned}
$$

where $G_{\mathrm{r}}$ and $G_{\mathrm{t}}$ are the reader and tag antenna gain, respectively, $\lambda$ is the wavelength and $L_{\mathrm{t}}$ accounts for the tag losses coming from the backscatter modulator, polarization 
mismatches and additional non-ideal effects. ${ }^{12}$ In fact, each tag is usually attached to a bigger object whose reflection properties, characterized by its RCS, could determine the presence of a strong clutter component having a TOA comparable to that of the useful signal.

In Fig. 6, the peaks of the received signal components are reported considering the reference scenario of Fig. 2, where 4 readers are placed at the corner of a $(7 \times 7) \mathrm{m}^{2}$ area. An object attached to the tag, with the RCS of a square metallic plate of dimensions $(30 \times 30) \mathrm{cm}^{2}$, is considered as scatter. The figure illustrates that the maximum received signal component is usually the one coming from the opposite transmitter. For this reason, a strong interference mitigation capability is requested for the de-spreading operation. In non-synchronous scenarios such a mitigation capability is hard to guarantee [21] and time division multiple access (TDMA) techniques between different transmitters have to be adopted to avoid interference, as discussed in Sec. V-A.

\section{B. Analog-to-Digital Conversion}

As previously stated, the receiver has to perform the signal de-spreading to detect and demodulate the tag signal. This process is generally performed in digital and, consequently, the ADC dynamic must be set to prevent saturation from the strongest signal components, that is, interference and clutter. In general, the receiver low-noise amplifier (LNA) must be designed to prevent saturation from the direct signals coming from other transmitters. It is important to underline that, as it will be described in Sec. V-B, in some cases the direct transmitter-to-receiver signals can be exploited for synchronization and integration of radar capabilities; in such cases, even if TDMA is adopted to handle the interferencefree access of different transmitters, a proper gain control has to be implemented to adapt the ADC dynamic to the input signals of different amplitude.

The number of quantization levels is then designed, as usual, for ensuring a satisfactory signal-to-quantization-noise ratio (SQNR) for the maximum allowed reader-tag distance (worst case). Note that the number of quantization bits is in general higher than that in the case of traditional one-way active communication links due to the two-hop channel and the corresponding poor link budget. In general, a multistatic configuration requires less bits for the ADC conversion, since the received tag signal experiences a lower dynamic for different receiver-tag distances. Fig. 7 presents the SQNR obtained at the output of the ADC whose maximum dynamic is adjusted considering the signal from the opposite transmitter in the reference square cell, as function of the reader-tag distance and for different number of quantization bits $m$. It is

\footnotetext{
${ }^{12}$ For UWB signals all the terms should be characterized as function of the frequency. This is a central-frequency approximation useful for understanding the order of magnitude of the signal level at receiver side. The first term $\mathrm{PL}_{i, k}^{(1,2)}$ accounts for the useful transmitter-tag-receiver backscattering information signal, or equivalently for the interfering component of a different tag. The term $\mathrm{PL}_{i, j}^{(3)}$ accounts for the direct path coming from an interfering transmitter, while $\mathrm{PL}_{i, k}^{(4)}$ for a clutter component, here approximated with the reflection from a scatter with a radar cross section (RCS) $\sigma$ placed in the tag's position.
}

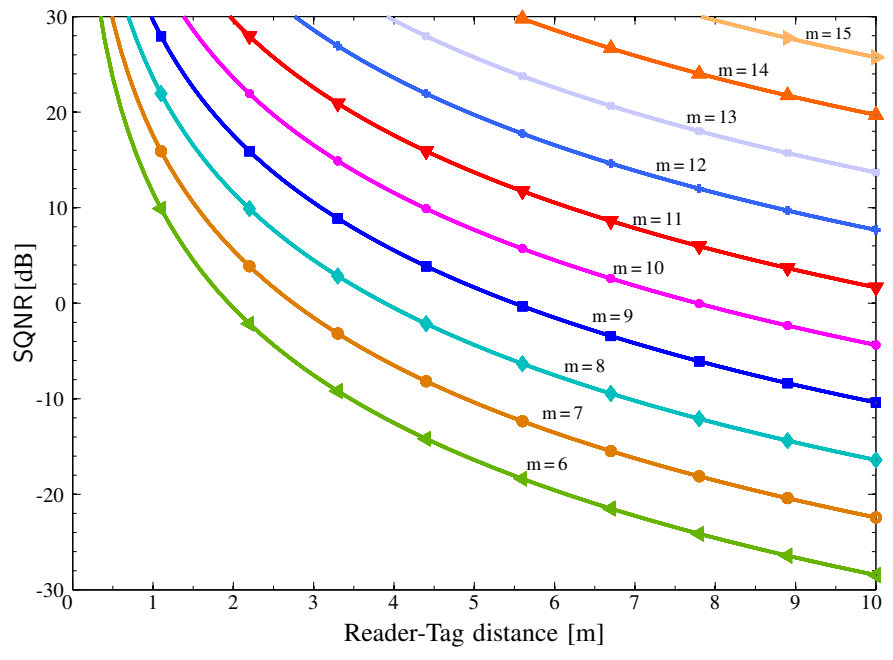

Fig. 7. SQNR at the ADC output.

immediate to observe how a high number of bits is necessary to provide a satisfactory SQNR so that the quantization noise is negligible with respect to the thermal noise (e.g., 14 bits for a SQNR of $20 \mathrm{~dB}$ at $9 \mathrm{~m}$ reader-tag distance). Fortunately, this requirement can be relaxed by considering the presence of the digital de-spreading process. In fact, in Appendix A it is shown that, in presence of low SNR conditions, as the case of the received UWB tag signal, the process gain is beneficial for increasing the effective SQNR, as $S Q N R_{\text {out }}=N_{\mathrm{s}} S Q N R_{\text {in }}$, where $S Q N R_{\text {in }}$ and $S Q N R_{\text {out }}$ refer to the $S Q N R$ at the input and at the output of the de-spreader, respectively. In this manner, the target SQNR at the output of the ADC can be reduced, resulting in a lower number of quantization bits. As an example, for a tag distance of $9 \mathrm{~m}$ with $N_{\mathrm{s}}=32768$, a SQNR out $_{\text {of }} 20 \mathrm{~dB}$ can be obtained with $\mathrm{SQNR}_{\text {in }}=-25 \mathrm{~dB}$, reflecting in the adoption of 7 bits instead of 15 .

An example of a receiver based on the scheme reported in [44], which prevents sampling at Nyquist rate, adopting 12 bits ADCs for the UWB-RFID reader is described in [24].

\section{NeTwORK DEPLOYMEnT}

In this section the challenges and the solutions concerning the multi-reader network deployment are presented.

\section{A. Multi-Reader Coexistence}

As already described, in the UWB-RFID network several readers monitor a certain area (four in the reference scenario of Fig. 2). It is then necessary to enable the possibility of accessing the same tag by multiple transmitters, with a potential problem of inter-reader interference.

For this analysis, the reference scenario reported in Fig. 2 is considered. As worst case, the presence of obstacles is neglected, assuming that the largest interference comes from the opposite transmitter in case of line-of-sight propagation. ${ }^{13}$ Without loss of generality, we focus on the interference

\footnotetext{
${ }^{13}$ In general it is possible to assume a lower level for the interference of the two neighbor readers in the case of partial directive antennas at transmitting and/or receiving stage, while a higher level for the interference coming from the two neighbor readers in case of adoption of omnidirectional antennas.
} 
generated by Reader 3 (opposite transmitter) on the Reader 1 , whose aim is to detect the tag presence by analyzing the backscattered response to its own transmitted signal. Thus, it is possible to foresee three different signal contributions:

1) The interfering signal of Reader 3 backscattered by the intended useful tag for Reader 1;

2) The direct path (and the multipath) between Reader 3 and Reader 1, responsible for the strongest interfering signal;

3) The interfering signal of Reader 3 backscattered by a tag different from the useful one.

1) CDMA Approach: If a CDMA-based technique is adopted for handling the multi-reader access, even a low residual interference component can completely vanish the possibility of detecting a tag signal. ${ }^{14}$ We assume that the interrogation signals transmitted by Reader 3 and Reader 1 are generated adopting the spreading codes $\left\{\tilde{d}_{n}^{(3)}\right\}$ and $\left\{\tilde{d}_{n}^{(1)}\right\}$, respectively, and that the useful and the interfering tags have codes $\left\{\tilde{c}_{n}^{(\mathrm{u})}\right\}$ and $\left\{\tilde{c}_{n}^{(\mathrm{int})}\right\}$, respectively. To detect the presence of the useful tag, Reader 1 performs a de-spreading using the composed code $\left\{\tilde{d}_{n}^{(1)} \cdot \tilde{c}_{n}^{(\mathrm{u})}\right\}$. In particular, relating to the previously presented three cases, the multi-reader interference is cancelled provided that the following three conditions are satisfied:

- Cancellation of the Reader 3 interference component modulated by the useful tag:

$$
\sum_{n=1}^{N_{\mathrm{s}}} \tilde{d}_{n}^{(1)} \tilde{c}_{n}^{(\mathrm{u})} \tilde{d}_{n}^{(3)} \tilde{c}_{n}^{(\mathrm{u})}=\sum_{n=1}^{N_{\mathrm{s}}} \tilde{d}_{n}^{(1)} \tilde{d}_{n}^{(3)}=0 .
$$

- Cancellation of the direct Reader 3-Reader 1 interference:

$$
\sum_{n=1}^{N_{\mathrm{s}}} \tilde{d}_{n}^{(1)} \tilde{c}_{n}^{(\mathrm{u})} \tilde{d}_{n}^{(3)}=0 .
$$

- Cancellation of the Reader 3 interference component modulated by the interfering tag:

$$
\sum_{n=1}^{N_{\mathrm{s}}} \tilde{d}_{n}^{(1)} \tilde{c}_{n}^{(\mathrm{u})} \tilde{d}_{n}^{(3)} \tilde{c}_{n}^{(\mathrm{int})}=0 .
$$

Note that condition (11) is satisfied adopting orthogonal codes and ensuring a tight synchronization between the readers (see Sec. V-B). Differently, (12) and (13) are substantially different from the classical conditions where good cross-correlation properties are required between pair of codewords [45], since here the products of three or four codewords are involved. These stringent requirements must be fulfilled for all the possible reader codes $d_{n}^{(j)}$ and all the possible tag codes $c_{n}^{(k)}$ (and their cyclic shifts due to the partial tag asynchronism) since it is necessary to remove the interference due to all the possible readers and tags. Due to the additional constraints on tag code properties [21], this poses several challenges on reader codes design, especially for the almost-ideal interference cancellation capability required.

\footnotetext{
${ }^{14}$ It can be shown that the difference between the power received by the opposite transmitter interference and that of a tag at $7 \mathrm{~m}$ from the reader is about $50 \mathrm{~dB}$.
}

2) TDMA Approach: For the aforementioned analysis, it is clear how the simultaneous interrogation of multiple transmitters is critical, and simpler solutions, such as TDMA, have to be accounted especially for low-complexity realizations. Specifically this consists on alternating in a cyclic way the transmitter, and setting the other readers in receiving mode. With the TDMA, the interference problem coming from other transmitters is completely avoided. TDMA can be performed at different rates considering the alternation of the transmitter, for example, each symbol or each packet. Decreasing the switching rate between transmitters (e.g., implementing TDMA at packet level) allows preventing problems deriving from synchronization mismatches, whereas the main drawback is the reduction of the refresh rate and constraints on the maximum tags' allowed speed, when tag tracking is performed. It is worthwhile to highlight that even the multi-reader access is TDMA-based, the multi-tag access is still CDMA-based.

3) Multistatic Configuration: An interesting alternative is represented by the adoption of a multistatic configuration with one only transmitter and at least 3 receivers [6]. In this manner the number of receivers is sufficient to provide unambiguous localization and the multi-transmitter interference problem is completely avoided. On the contrary, with this configuration, the diversity provided by the signals from more than one transmitter is no more available, and it is fundamental to guarantee the perfect visibility of the tag antenna with the transmitter, avoiding non-line-of-sight (NLOS) conditions.

\section{B. Reader Synchronization}

Readers must be kept synchronized in order to ensure satisfactory multiple access performance and to allow multistatic functionalities. Coarse synchronization can be provided with the reader-central unit links (e.g., realized with a standard Ethernet protocol). Fine synchronization can exploit the same UWB interrogation signals emitted by transmitters, hence reusing the same hardware developed for tag detection. In fact, the lower path loss of the direct transmitter-to-receiver link ensures a very high SNR for the demodulation of the transmitter signal (static contribution), allowing very accurate (subnanosecond) TOA estimation. In this case, the de-spreading is operated according to the incoming transmitter code $\tilde{d}_{n}^{(j)}$ only, without accounting for the tag code. Since the transmitterreceiver distance is fixed, TOA estimation can be compared with the expected propagation time between the two antennas, adjusting consequently the reader's clock according to the difference between the estimated and expected range. The process can be further iterated until the difference in the clock adjustment falls below a threshold, indicating the reached synchronism.

Summarizing, the synchronization process is enabled through two facts: (i) transmitters are placed in known positions; (ii) de-spreading operation is conducted exploiting the transmitters' code $\left\{\tilde{d}_{n}^{(j)}\right\}$ only, resulting in the isolation of the static contribution $r_{i, j}^{\mathcal{S}}(t)$ at receiver side. Specifically, by performing TOA estimation on the signal $r_{i, j}^{\mathcal{S}}(t)$, the $i$ th receiver estimates $\widehat{\tau}_{i, j}^{\prime}=\widehat{\tau}_{i, j}+T_{i, j}$, where $T_{i, j}$ is the unknown clock offset between the $j$ th transmitter and the 
$i$ th receiver. Since transmitters' and receivers' positions are known, the true distance $d_{i, j}=\left|\mathbf{p}_{\mathrm{T} j}-\mathbf{p}_{\mathrm{R} i}\right|$ between the transmitter and the receiver composing the bistatic pair can be exploited to determine $T_{i, j}$ and synchronize the receiver with the transmitter.

\section{Tag Localization}

After the initial reader synchronization step, a second despreading operation conducted with the composed code $\left\{\tilde{d}_{n}^{(j)}\right.$. $\left.\tilde{c}_{n}^{(k)}\right\}$ allows isolating the $k$ th tag component $r_{i, j}^{\mathcal{T}}(t)$. Now, TOA estimation of such a signal gives $\hat{\tau}_{i, j, k}$, and consequently $\hat{d}_{i, j, k}=c \hat{\tau}_{i, j, k}$, which corresponds to the estimation of the sum $d_{\mathrm{T} j}+d_{\mathrm{R} i}$. The term $\hat{d}_{i, j, k}$ can be exploited to define the ellipse related to the bistatic pair of interest, with the foci located in the considered transmitter and receiver. With the intersection of several ellipses the tag's position is then determined [46], as usually realized in radar networks where transmitters and receivers are often not co-located. Notice that, when a monostatic configuration is adopted, the receiver is intrinsically synchronous with the co-located transmitter, and the reader-tag distance is directly computed from the signal round-trip time (RTT). In this case localization is simply realized with the intersection of circumferences [3].

Fig. 8 presents the localization performance in terms of complementary cumulative distribution function (CCDF) of the localization error in the reference scenario of Fig. 2, when TOA estimation is performed with an energy detector with optimum threshold [10]. A monostatic configuration is considered, and the TOA estimates collected at the three readers receiving the greatest amount of power from the tag were selected as input of a standard least squares localization algorithm [3]. Simulation accounts for $10^{5}$ static random test positions. It is evident that by increasing the number of pulses per symbol $N_{\mathrm{s}}$ the localization performance is improved, thanks to the additional process gain determining more accurate TOA estimates. Adopting $N_{\mathrm{s}}=32768$, a localization error below $10 \mathrm{~cm}$ is guarantee for the $90 \%$ of the tag positions in the reference scenario, making the solution very appealing for practical applications. Recently, performance bounds for the localization accuracy in both monostatic and multistatic RFID configurations have been derived [6], [47], highlighting the role of system parameters, network configuration and topology.

\section{An Alternative Approach: Hybrid UHF-UWB TAGS}

In the previous sections, it has been shown that even if the passive UWB-RFID architecture is very promising, it might suffer from several issues that can increase the system complexity. Thus, in this section, a new appealing solution for IoT applications is introduced. The idea is based on the integration of the previously described UWB tag with a standard UHF EPC Gen.2 tag.

Consider a typical supply chain scenario, where these proposed hybrid RFID tags are applied on goods which travel inside the factory. Tag identification can be normally realized with the UHF Gen.2 readers, exploiting the compatibility of the tags with the standard protocol. When goods enter in

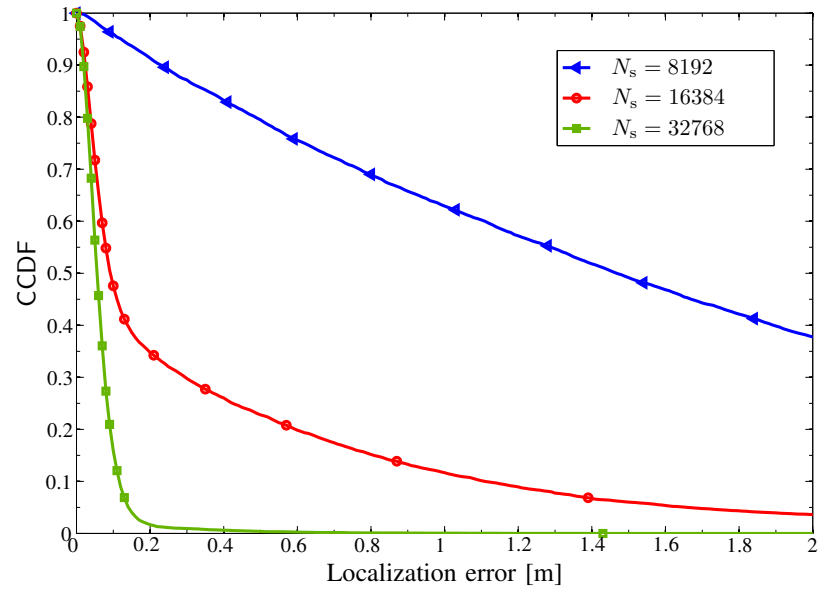

Fig. 8. Complementary cumulative distribution function (CCDF) of the localization error for different number of pulses per symbol.

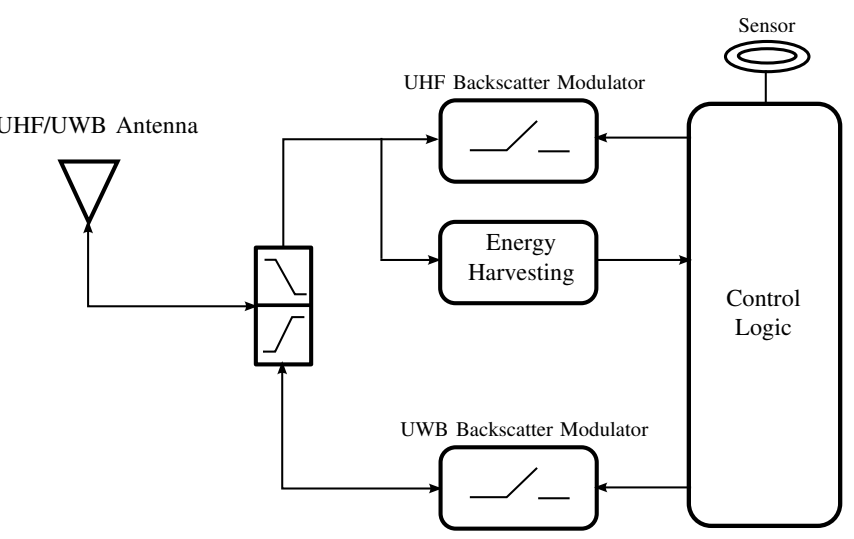

Fig. 9. Hybrid UHF-UWB tag.

a critical area where high-accuracy location information is fundamental, for example in a section of a conveyor belt where they need to be correctly sorted (see Fig. 1) [48]-[50], the additional UWB interface can fulfill the high-accuracy localization requirement. Fig. 9 presents the scheme of the hybrid UHF-UWB tag, which offers the important advantage of resulting compatible with the state-of-the-art Gen.2 tag. The UWB interface can be viewed as an add-on allowing accurate tag localization, which represents an extremely appealing feature for practical applications. In the following, two possible architectures exploiting these hybrid tags are discussed.

\section{A. Option 1: UHF-UWB Portals}

The first architectural solution for the exploitation of the hybrid UHF-UWB tags consists in the adoption of a UHF portal placed at the entrance of the area where localization becomes critical. Such a portal guarantees a log-in phase of the hybrid tags that are registered in the critical area. However, differently from the stand-alone UWB tags previously described, the portal assigns a dedicated code $c_{n}^{(k)}$ to each tag by exploiting the Gen.2 link communication capability. Such code is successively adopted by the UWB-RFID network for providing simultaneous localization of multiple tags inside that critical area only. In this manner, the few available codes can 
be reused in several separated areas, managed by different portals, improving the system efficiency and avoiding the need of a unique spreading code per tag. Moreover, the standard Gen.2 tag-reader link can be used also for data communication (i.e., to transfer sensor data from the tag). In this case, there is no need of exploiting the UWB tag-reader link for data communication, and a single bit in the UWB packet, that is, $N_{\mathrm{p}}=1$, is sufficient for tag ranging (i.e., only $N_{\mathrm{s}}$ pulses per tag are sent and backscattered). In this way, the specifications on the tag local oscillator can be relaxed, as well as the clock drift problem will not impact the short transmitted packet [25]. Since now only one bit is transmitted, the number of pulses $N_{\mathrm{s}}$ can be significantly enlarged to provide improved detection range and accurate TOA estimation thanks to the increased process gain. Such an architectural solution ensures high refresh rates since the simultaneous CDMA of tags is exploited, but maintains the intrinsic problems discussed in the previous sections of the UWB-RFID, such as multi-reader and multi-tag interference. The last effect can be partially mitigated by the exploitation of several portals with only few tags simultaneously active. It is important to underline that the tag power consumption is increased due to the presence of the Gen.2 UHF circuitry with respect to the stand-alone UWB tag.

\section{B. Option 2: Tight UHF-UWB Interaction}

This second architectural solution is capable of overcoming the multi-tag interference problem, as it is completely managed by the standard Gen.2 RFID. The idea is to introduce a tight interaction between the UHF and the UWB protocols [51]. In this case, the UHF reader broadcasts an addressing command for a specific tag with the Gen.2 signaling. All the tags demodulate such UHF Gen.2 signal, but only the specific tag addressed activates its UWB backscatter modulator. In such a manner, only one UWB tag is active in each interrogation and, consequently, the multi-tag interference is completely avoided. In addition, the receiver structure is enormously simplified, as only one code is shared among all tags without the need of replicating the receiver structure. Thus, differently from the adoption of the stand-alone passive UWB-RFID, the UHFUWB system has no limitations in terms of number of manageable tags. As for the previous option, data communication can be performed with the UHF link in order to maintain a short UWB packet and increase the number of pulses per symbol. Finally, this alternative solution is beneficial also for the multi-reader deployment. In fact, conditions (11), (12), (13) necessary for avoiding multi-reader interference are easily satisfied since, with the adoption of this architecture, only one tag backscatters the incoming reader signals. Consequently, good cross-correlation properties are easier to find with a single tag codeword.

These solutions let to merge most of the benefits of both UHF and UWB RFID schemes, by maintaining a substantial low system complexity.

\section{CONCLUSION}

In this work, the system design of a UWB-RFID network for tag localization in IoT applications has been presented.

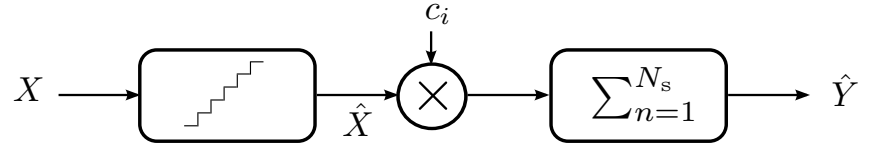

Fig. 10. The considered scheme for the ADC and de-spreader.

The main available results from the literature have been revised, highlighting the challenging issues and proposing new solutions. Aspects usually separately investigated such as signaling schemes, signal processing, receiver design and multireader network deployment have been presented in a unitary form, addressing the impact of each choice on the system design. In addition, new solutions based on the integration of the UWB-RFID with the current UHF-RFID technology have been proposed and discussed. It has been shown as the architectural choice is strictly application-dependent, and must account for costs, complexity, energy efficiency, backward compatibility and performance. All the problems and solutions herein addressed can drive the design of passive UWB-RFID systems, which represent an interesting and effective candidate for the paradigm of the IoT when tag localization is a key requirement.

\section{ACKNOLEDGMENT}

The authors would like to thank all the consortium of the FP7 SELECT project and GRETA project, A. Guerra and R. D'Errico for the fruitful cooperation.

\section{APPENDIX}

It is here derived the relationship between the SQNR at the output of the ADC and of the de-spreader as a function of the number of pulses $N_{\mathrm{s}}$, as reported in Sec. IV-B. Fig. 10 shows the analyzed ADC and de-spreader scheme, where the ADC output is multiplied by the tag code, and then it is accumulated for $N_{\mathrm{s}}$ times. In particular, it is possible to express the input signal $X$ as

$$
X=x_{\mathrm{u}}+x_{\mathrm{c}}+n_{i}
$$

where $x_{\mathrm{u}}$ is the useful signal component, $x_{\mathrm{c}}$ is the clutter/interference component (which mainly affects the dynamic range at the $\mathrm{ADC}$ input), and $n_{i}$ is the additive thermal noise. The useful signal component and the clutter component are assumed uniformly distributed respectively in $\left[X^{\min }, X^{\max }\right]$ and $\left[-X_{\mathrm{c}}^{\min }, X_{\mathrm{c}}^{\max }\right]$, and are considered both constant within a symbol time.

It is possible to express the quantized version $\hat{X}$ of $X$ as

$$
\hat{X}=x_{\mathrm{u}}+x_{\mathrm{c}}+n_{i}+\epsilon_{x}
$$

where $\epsilon_{x}$ is the quantization noise error. Assume the quantization noise uniformly distributed in $[0, \delta]$, where $\delta$ corresponds to the quantization step amplitude. ${ }^{15}$ Now, looking at the output of the accumulator, it is possible to express $\hat{Y}$, under the hypothesis of zero mean code, as

$$
\hat{Y}=N_{\mathrm{s}} x_{\mathrm{u}}+n_{\text {out }}+\epsilon_{y}
$$

${ }^{15}$ Assuming that a sufficient number of quantization bits is adopted and that the sum of signal and noise is above the quantization step. 
where $n_{\text {out }}$ is given by $n_{\text {out }}=\sum_{i=1}^{N_{\mathrm{s}}} c_{i} n_{i}$, and $c_{i}$ represents the $i$ th value of the code $\tilde{c}_{n}^{(k)}$.

The SQNR SQNR in and SQNR ${ }_{\text {out }}$ at the output of the ADC and of the accumulator, respectively, are

$$
\mathrm{SQNR}_{\text {in }}=\frac{\mathbb{E}\left\{x_{\mathrm{u}}^{2}\right\}}{\mathbb{E}\left\{\epsilon_{x}^{2}\right\}}
$$

and

$$
\mathrm{SQNR}_{\text {out }}=\frac{N_{\mathrm{s}}^{2} \mathbb{E}\left\{x_{\mathrm{u}}^{2}\right\}}{\mathbb{E}\left\{\epsilon_{y}^{2}\right\}}
$$

where $\mathbb{E}\{Z\}$ is the expected value of the random variable $Z$. In the following, the relationship between $\mathbb{E}\left\{\epsilon_{y}^{2}\right\}$ and $\mathbb{E}\left\{\epsilon_{x}^{2}\right\}$ is derived. In particular, it is possible to write the second-order moment of $\epsilon_{y}$ as

$$
\begin{aligned}
\mathbb{E}\left\{\epsilon_{y}^{2}\right\} & =\mathbb{E}\left\{\left(\sum_{i=1}^{N_{\mathrm{s}}} c_{i} \epsilon_{x_{i}}\right)^{2}\right\} \\
& =\mathbb{E}\left\{\sum_{i=1}^{N_{\mathrm{s}}} c_{i}^{2} \epsilon_{x_{i}}^{2}+2 \sum_{i=1}^{N_{\mathrm{s}}-1} \sum_{j=i+1}^{N_{\mathrm{s}}} c_{i} \epsilon_{x_{i}} c_{j} \epsilon_{x_{j}}\right\} .
\end{aligned}
$$

Expression (19) is now made particular in the low SNR case, where the thermal noise amplitude is larger than the quantization step and the useful signal amplitude, which is the condition of interest for the UWB-RFID system. In this case, it is possible to assume that $\epsilon_{x_{i}}$ and $\epsilon_{x_{j}}$ are independent (due to the Gaussian thermal noise), obtaining

$$
\mathbb{E}\left\{2 \sum_{i=1}^{N_{\mathrm{s}}-1} \sum_{j=i+1}^{N_{\mathrm{s}}} c_{i} \epsilon_{x_{i}} c_{j} \epsilon_{x_{j}}\right\}=0 .
$$

As a consequence, it is possible to write

$$
\mathbb{E}\left\{\epsilon_{y}^{2}\right\}=\mathbb{E}\left\{\sum_{i=1}^{N_{\mathrm{s}}} c_{i}^{2} \epsilon_{x_{i}}^{2}\right\}=\sum_{i=1}^{N_{\mathrm{s}}} c_{i}^{2} \mathbb{E}\left\{\epsilon_{x_{i}}^{2}\right\}=N_{\mathrm{s}} \mathbb{E}\left\{\epsilon_{x}^{2}\right\}
$$

which gives, from (18), $\mathrm{SQNR}_{\text {out }}=N_{\mathrm{S}} \mathrm{SQNR}_{\text {in }}$, showing the process gain of $N_{\mathrm{S}}$ for the SQNR introduced by the despreading process.

\section{REFERENCES}

[1] C. Floerkemeier et al., Eds., The Internet of Things, First International Conference. Springer, 2008.

[2] D. Zhang et al., "Real-time locating systems using active RFID for Internet of Things," IEEE Syst. J., no. 99, pp. 1-10, 2014, early Access Online.

[3] D. Dardari, E. Falletti, and M. Luise, Satellite and Terrestrial Radio Positioning Techniques - A signal processing perspective. Elsevier Ltd, London, 2011.

[4] K. Finkenzeller, RFID Handbook: Fundamentals and Applications in Contactless Smart Cards and Identification., 3rd ed. Wiley, 2010.

[5] P. Nikitin and K. Rao, "Theory and measurement of backscattering from RFID tags," IEEE Antennas Propag. Mag., vol. 48, no. 6, pp. 212-218, Dec. 2006.

[6] N. Decarli, F. Guidi, and D. Dardari, "A novel joint RFID and radar sensor network for passive localization: Design and performance bounds," IEEE J. Sel. Topics Signal Process., vol. 8, no. 1, pp. 80-95, Feb. 2014.

[7] V. Viikari, P. Pursula, and K. Jaakkola, "Ranging of UHF RFID tag using stepped frequency read-out," IEEE Sensors J., vol. 10, no. 9, pp. 1535-1539, Sep. 2010.

[8] A. Costanzo et al., "Tag, you're it: Ranging and finding via RFID technology," IEEE Microw. Mag., vol. 14, no. 5, pp. 36-46, Aug. 2013.
[9] S. Shao and R. Burkholder, "Item-level RFID tag location sensing utilizing reader antenna spatial diversity," IEEE Sensors J., vol. 13, no. 10 , pp. 3767-3774, Oct. 2013.

[10] D. Dardari et al., "Ranging with ultrawide bandwidth signals in multipath environments," Proc. IEEE, vol. 97, no. 2, pp. 404-426, Feb. 2009.

[11] Y. Shen and M. Z. Win, "Fundamental limits of wideband localization Part I: A general framework," IEEE Trans. Inf. Theory, vol. 56, no. 10, pp. 4956-4980, Oct. 2010.

[12] D. Dardari et al., "Ultrawide bandwidth RFID: The next generation?" Proc. IEEE, vol. 98, no. 9, pp. 1570-1582, Sep. 2010.

[13] A. Ramos et al., "Solar-powered wireless temperature sensor based on UWB RFID with self-calibration," IEEE Sensors J., vol. 15, no. 7, pp. 3764-3772, Jul. 2015.

[14] B. Sobhani et al., "Target tracking for UWB multistatic radar sensor networks," IEEE J. Sel. Topics in Signal Process., vol. 8, no. 1, pp. 1932-4553, Feb. 2014.

[15] S. Bartoletti et al., "Sensor radar networks for indoor tracking," IEEE Wireless Commun. Lett., vol. 3, no. 2, pp. 157-160, Apr. 2014.

[16] D. Arnitz, U. Muehlmann, and K. Witrisal, "UWB ranging in passive UHF RFID: Proof of concept," IEEE Electronics Lett., vol. 46, no. 20, pp. 1401-1402, Oct. 2010.

[17] M. Baghaei-Nejad et al., "A remote-powered RFID tag with $10 \mathrm{Mb} / \mathrm{s}$ UWB uplink and $18.5 \mathrm{dBm}$ sensitivity UHF downlink in 0.18 CMOS," in IEEE Int. Solid-State Circuits Conference (ISSCC), Feb. 2009, pp. 198-199.

[18] D. Dardari, "Pseudo-random active UWB reflectors for accurate ranging," IEEE Commun. Lett., vol. 8, no. 10, pp. 608-610, Oct. 2004.

[19] A. Lazaro et al., "Active UWB reflector for RFID and wireless sensor networks," IEEE Trans. Antennas Propag., vol. 61, no. 9, pp. 47674774, Sep. 2013.

[20] F. Guidi et al., "Analysis of UWB tag backscattering and its impact on the detection coverage," IEEE Trans. Antennas Propag., vol. 62, no. 8, pp. 4292-4303, Aug. 2014.

[21] — "Detection of multiple tags based on impulsive backscattered signals," IEEE Trans. Commun., vol. 62, no. 11, pp. 3918-3930, Nov. 2014.

[22] A. Ramos, A. Lazaro, and D. Girbau, "Semi-passive time-domain UWB RFID system," IEEE Trans. Microwave Theory Tech., vol. 61, no. 4, pp. 1700-1708, Apr. 2013.

[23] V. Heiries et al., "UWB backscattering system for passive RFID tag ranging and tracking," in Proc. IEEE Int. Conf. on Ultra-Wideband (ICUWB), Bologna, Italy, Sep. 2011, pp. 489-493.

[24] E. Savioli et al., "Semi-passive UHF-UWB RFID: Architecture and localization performance," in Proc. IEEE Int. Conf. on Commun. Workshops, Budapest, Hungary, Jun. 2013, pp. 1-5.

[25] N. Decarli et al., "Interference and clock drift effects in UWB RFID systems using backscatter modulation," in Proc. IEEE Int. Conf. on Ultra-Wideband (ICUWB), Syracuse, NY, Sep. 2012, pp. 1-5.

[26] S. Hu et al., "Detection range enhancement of UWB RFID systems," in IEEE Int. Workshop on Anti-counterfeiting, Security, Identification, Xiamen, China, Apr. 2007, pp. 431-434.

[27] J. Kimionis, A. Bletsas, and J. N. Sahalos, "Increased range bistatic scatter radio," IEEE Trans. Commun., vol. 62, no. 3, pp. 1091-1104, Mar. 2014.

[28] A. Guerra et al., "Energy sprinklers for passive UWB RFID," in Proc. IEEE Int. Conf. on Ultra-Wideband (ICUWB), Paris, France, Sep. 2014, pp. 1-6.

[29] S. Hu, C. Law, and W. Dou, "Measurements of UWB antennas backscattering characteristics for RFID systems," in Proc. IEEE Int. Conf. on Ultra-Wideband (ICUWB), Singapore, Sep. 2007, pp. 94-99.

[30] _ _ "A balloon-shaped monopole antenna for passive UWB-RFID tag applications," IEEE Antennas Wireless Propag. Lett., vol. 7, pp. 366368, 2008.

[31] M. Fantuzzi, D. Masotti, and A. Costanzo, "A novel integrated UWBUHF one-port antenna for localization and energy harvesting," IEEE Trans. Antennas Propag., vol. 63, no. 9, pp. 3839-3848, Sep. 2015.

[32] D. Dardari et al., "Ultra-wide bandwidth backscatter modulation: Processing schemes and performance," EURASIP J. Wireless Commun. Networking, pp. 1-15, Jul. 2011.

[33] P. Meissner, E. Leitinger, and K. Witrisal, "UWB for robust indoor tracking: Weighting of multipath components for efficient estimation," IEEE Wireless Commun. Lett., vol. 3, no. 5, pp. 501-504, Oct. 2014.

[34] "IEEE standard for information technology - telecommunications and information exchange between systems - local and metropolitan area networks - specific requirement part 15.4: Wireless medium access control (MAC) and physical layer (PHY) specifications for low-rate 
wireless personal area networks (WPANs)," IEEE Std 802.15.4a-2007 (Amendment to IEEE Std 802.15.4-2006), pp. 1-203, 2007.

[35] R. D'Errico, “An indoor backscattering channel characterization for UWB passive RFID applications," in European Conf. on Antennas and Propag. (EuCAP), Prague, Czech Republic, Mar. 2012, pp. 1169-1173.

[36] R. D'Errico, J. Keignart, and L. Rudant, "Characterization of UWB backscattering propagation for passive tags identification and localization," in European Conf. on Antennas and Propag. (EuCAP), Gothenburg, Sweden, Apr. 2013, pp. 1909-1913.

[37] A. Sibille et al., "Channel modeling for backscattering based UWB tags in a RTLS system with multiple readers," in European Conf. on Antennas and Propag. (EuCAP), Gothenburg, Sweden, Apr. 2013, pp. 3022-3026.

[38] J. Griffin and G. Durgin, "Complete link budgets for backscatter-radio and RFID systems," IEEE Antennas Propag. Mag., vol. 51, no. 2, pp. 11-25, Apr. 2009.

[39] F. Guidi, A. Sibille, and C. Roblin, "Interaction of UWB tag backscattering with close metallic reflectors," IEEE Antennas Wireless Propag. Lett., vol. 13, pp. 245-248, 2014.

[40] R. D'Errico et al. "An UWB-UHF semi-passive RFID system for localization and tracking applications," in Proc. IEEE Int. Conf. on RFIDTechnology and Applications (RFID-TA), Nice, France, Nov. 2012, pp. $1-6$.

[41] K. K. Lee, K. Granhaug, and N. Andersen, "A study of low-power crystal oscillator design," in Proc. NORCHIP Conf., Vilnius, Lithuania, Nov. 2013, pp. $1-4$

[42] "EPC Radio-Frequency Identity Protocols Class-1 Generation-2 UHF RFID," EPC global 2004-2008 Inc., pp. 1-108, 2008.

[43] P. Di Marco, F. Santucci, and C. Fischione, "Modeling anti-collision protocols for RFID systems with multiple access interference," in Proc. IEEE Int. Conf. on Commun., Sydney, Australia, Jun. 2014, pp. 59385944.

[44] D. Lachartre et al., "A $1.1 \mathrm{~nJ} / \mathrm{b}$ 802.15.4a-compliant fully integrated UWB transceiver in $0.13 \mu \mathrm{m} \mathrm{CMOS,"} \mathrm{in} \mathrm{Proc.} \mathrm{of} \mathrm{the} \mathrm{ISSCC,} \mathrm{San}$ Francisco, USA, Feb. 2009.

[45] D. Sarwate and M. Pursley, "Crosscorrelation properties of pseudorandom and related sequences," Proc. IEEE, vol. 68, no. 5, pp. 593-619, May 1980.

[46] M. Malanowski and K. Kulpa, "Two methods for target localization in multistatic passive radar," IEEE Trans. Aerospace Electronic Syst., vol. 48, no. 1, pp. 572-580, Jan. 2012.

[47] N. Decarli and D. Dardari, "RFID and Radar localization: A position error bound analysis," in Proc. IEEE Int. Conf. on Commun. Workshops, Budapest, Hungary, Jun. 2013, pp. 37-41.

[48] S. Bartoletti et al., "Order of arrival estimation via UHF-UWB RFID," in Proc. IEEE Int. Conf. on Commun. Workshops, Sydney, Australia, Jun. 2014, pp. 133-137.

[49] A. Buffi, P. Nepa, and F. Lombardini, "A phase-based technique for localization of UHF-RFID tags moving on a conveyor belt: Performance analysis and test-case measurements," IEEE Sensors J., vol. 15, no. 1, pp. 387-396, Jan. 2015.

[50] L. Shangguan et al., "OTrack: Towards order tracking for tags in mobile RFID system," IEEE Trans. Parallel Distrib. Syst., vol. 25, no. 8, pp. 2114-2125, Aug. 2013.

[51] P. Di Marco et al., "An UWB-enhanced identification procedure for large-scale passive RFID systems," in Proc. IEEE Int. Conf. on UltraWideband (ICUWB), Paris, France, Sep. 2014, pp. 421-426.

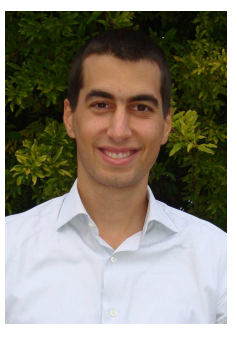

Nicolò Decarli (S'10-M'14) received the B.S. degree and the M.S. degree, in telecommunications engineering and with honors, from the University of Bologna, Italy, in 2007 and 2009, respectively. In 2013 he received the $\mathrm{PhD}$ degree in electronics, telecommunications and information technologies.

$\mathrm{He}$ is currently a postdoctoral researcher at DEI, University of Bologna, Italy. In 2012, he spent a year as visiting student of the Wireless Communication and Network Sciences Laboratory at the Massachusetts Institute of Technology (MIT). His research interests include ultra-wide bandwidth communications systems, radio localization, wireless sensor networks and RFID.

Dr. Decarli serves as reviewer for numerous IEEE Journals and Conferences. He was a member of the local organization committee for the 2011 IEEE International Conference on Ultra-WideBand and TPC member of the IEEE ICC 2015 Workshop on Advances in Network Localization and Navigation (ANLN).

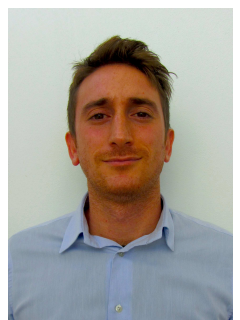

Francesco Guidi (S'09 - M'14) received the B.S. degree and the M.S. degree (both summa cum laude) from the University of Bologna in biomedical (2006) and in electronics and telecommunications (2009) engineering, respectively. In 2013 he received the $\mathrm{PhD}$ degree both from Ecole Polytechnique ParisTech, France (Computer Science Specialty) and from University of Bologna, Italy, in electronics, telecommunications and information technologies.

$\mathrm{He}$ is currently a Marie Curie Fellow at CEALETI, Grenoble, France. From mid-2013 to mid2015 he was a postdoctoral researcher at University of Bologna. His research interests include RFID technology, joint antenna and channel characterization, signal processing, UWB and mm-waves technologies. He was the recipient of the best student paper award at the 2014 IEEE International Conference on Ultra-Wideband. Dr. Guidi serves as reviewer for numerous IEEE Journals and Conferences and was a member of the local organization committee for the 2011 IEEE International Conference on Ultra-Wideband and TPC member of the IEEE ICC 2015 ANLN Workshop and of IEEE Globecom 2015 LION Workshop.

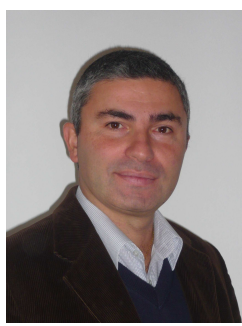

Davide Dardari (SM'07) is an Associate Professor at the University of Bologna, Italy, where he participates with WiLAB (Wireless Communications Laboratory). Since 2005, he has been a Research Affiliate at Massachusetts Institute of Technology (MIT), Cambridge, USA. His research interests involve ultra-wide bandwidth (UWB) systems, network localization, passive communication systems, distributed estimation theory and wireless sensor networks. He received the IEEE Aerospace and Electronic Systems Societys M. Barry Carlton Award (2011) and the 2012 IEEE Communications Society Fred W. Ellersick Prize. Prof. Dardari is Senior Member of the IEEE where he served as Chair for the Radio Communications Committee of the IEEE Communication Society. He was co-General Chair of the 2011 IEEE International Conference on UltraWideband, and TPC Chair of several conferences. He served as an Editor for IEEE Transactions on Wireless Communications from 2006 to 2012 and Guest Editor for several special issues. 